\title{
Gisin's Theorem for Three Qubits
}

\author{
Jing-Ling Chen,,${ }^{1,2, *}$ Chunfeng $\mathrm{Wu},{ }^{1}$ L. C. Kwek,,${ }^{1,3}$ and C. H. Oh ${ }^{1, 甘}$ \\ ${ }^{1}$ Department of Physics, National University of Singapore, 2 Science Drive 3, Singapore 117542 \\ ${ }^{2}$ Institute of Applied Physics and Computational Mathematics, P.O. Box 8009 (26), 100088 Beijing, China \\ ${ }^{3}$ Nanyang Technological University, National Institute of Education, 1, Nanyang Walk, Singapore 637616
}

We present a Theorem that all generalized Greenberger-Horne-Zeilinger states of a three-qubit system violate a Bell inequality in terms of probabilities. All pure entangled states of a three-qubit system are shown to violate a Bell inequality for probabilities; thus, one has Gisin's theorem for three qubits.

PACS numbers: 03.65.Ud, 03.67.Mn,42.50.-p

Quantum mechanics violates Bell type inequalities that hold for any local-realistic theory [1, 2, 3, 4, 5]. In 1991, Gisin presented a theorem, which states that any pure entangled state of two particles violates a Bell inequality for two-particle correlation functions [6, 7]. Bell's inequalities for systems of more than two qubits are the object of renewed interest, motivated by the fact that entanglement between more than two quantum systems is becoming experimentally feasible. Recent investigations show the surprising result that there exists a family of pure entangled $N>2$ qubit states that do not violate any Bell inequality for $N$-particle correlations for the case of a standard Bell experiment on $N$ qubits 8 . By a standard Bell experiment we mean the one in which each local observer is given a choice between two dichotomic observables $9,10,11,12$. This family is the generalized Greenberger-Horne-Zeilinger (GHZ) states given by

$$
|\psi\rangle_{\mathrm{GHZ}}=\cos \xi|0 \cdots 0\rangle+\sin \xi|1 \cdots 1\rangle
$$

with $0 \leq \xi \leq \pi / 4$. The GHZ states [3] are for $\xi=\pi / 4$. In 2001, Scarani and Gisin noticed that for $\sin 2 \xi \leq 1 / \sqrt{2^{N-1}}$ the states (11) do not violate the Mermin-Ardehali-Belinskii-Klyshko (MABK) inequalities. Based on which, Scarani and Gisin wrote that "this analysis suggests that MK [in Ref. [9], MABK] inequalities, and more generally the family of Bell's inequalities with two observables per qubit, may not be the 'natural' generalizations of the CHSH inequality to more than two qubits" [8], where CHSH stands for Clauser-Horne-Shimony-Holt. In Ref. [10] Żukowski and Brukner (ŻB) have derived a general Bell inequality for correlation functions for $N$ qubits. The ŻB inequalities include MABK inequalities as special cases. Ref. [9] shows that (a) For $N=$ even, although the generalized GHZ state (11) does not violate MABK inequalities, it violates the ŻB inequality and (b) For $\sin 2 \xi \leq 1 / \sqrt{2^{N-1}}$ and $N=$ odd, the correlations between measurements on qubits in the generalized GHZ state (1) satisfy all Bell inequalities for correlation functions, which involve two dichotomic observables per local measurement station.

In this Letter, we focus on a three-qubit system, whose corresponding generalized GHZ state reads $|\psi\rangle_{\mathrm{GHZ}}=$ $\cos \xi|000\rangle+\sin \xi|111\rangle$. Up to now, there is no Bell inequality violated by this pure entangled state for the region $\xi \in(0, \pi / 12]$ based on the standard Bell experiment. Can Gisin's theorem be generalized to three-qubit pure entangled states? Can one find a Bell inequality that violates $|\psi\rangle_{\mathrm{GHZ}}$ for the whole region? In the following, we first present a theorem that all generalized GHZ states of a three-qubit system violate a Bell inequality in terms of probabilities; second, we will provide a universal Bell inequality for probabilities that is violated by all pure entangled states of a three-qubit system.

Theorem 1: All generalized GHZ states of a three-qubit system violate a Bell inequality for probabilities.

Proof: Let us consider the following Bell-type scenario: three space-separated observers, denoted by $A, B$ and $C$ (or Alice, Bob and Charlie), can measure two different local observables of two outcomes, labelled by 0 and 1 . We denote $X_{i}$ the observable measured by party $X$ and $x_{i}$ the outcome with $X=A, B, C(x=a, b, c)$. If the observers decide to measure $A_{1}, B_{1}$ and $C_{2}$, the result is $(0,1,1)$ with probability $P\left(a_{1}=0, b_{1}=1, c_{2}=1\right)$. The set of these $8 \times 8$ probabilities gives a complete description of any statistical quantity that can be observed in this Gedanken experiment. One can easily see that, any local-realistic (LR) description of the previous Gedanken experiment satisfies the following Bell inequality:

$$
\begin{aligned}
& P\left(a_{1}+b_{1}+c_{1}=0\right)+P\left(a_{1}+b_{1}+c_{1}=3\right)+P\left(a_{1}+b_{2}+c_{2}=2\right) \\
& +P\left(a_{2}+b_{1}+c_{1}=0\right)+P\left(a_{2}+b_{1}+c_{1}=3\right)+P\left(a_{2}+b_{2}+c_{2}=1\right) \\
& -P\left(a_{1}+b_{1}+c_{1}=1\right)-P\left(a_{1}+b_{2}+c_{2}=1\right) \\
& -P\left(a_{2}+b_{1}+c_{1}=2\right)-P\left(a_{2}+b_{2}+c_{2}=2\right) \leq 2 .
\end{aligned}
$$


The joint probability $P\left(a_{i}+b_{j}+c_{k}=r\right)$, for instance, $P\left(a_{i}+b_{j}+c_{k}=1\right)=P\left(a_{i}=1, b_{j}=0, c_{k}=0\right)+P\left(a_{i}=\right.$ $\left.0, b_{j}=1, c_{k}=0\right)+P\left(a_{i}=0, b_{j}=0, c_{k}=1\right)$. However, quantum mechanics will violate this Bell inequality for any generalized GHZ states. The quantum prediction for the joint probability reads

$$
P^{Q M}\left(a_{i}=m, b_{j}=n, c_{k}=l\right)=\left\langle\psi\left|\hat{P}\left(a_{i}=m\right) \otimes \hat{P}\left(b_{j}=n\right) \otimes \hat{P}\left(c_{k}=l\right)\right| \psi\right\rangle
$$

where $i, j, k=0,1 ; m, n, l=0,1$, and

$$
\begin{aligned}
& \hat{P}\left(a_{i}=m\right)=\frac{1+(-1)^{m} \hat{n}_{a_{i}} \cdot \vec{\sigma}}{2} \\
& =\frac{1}{2}\left(\begin{array}{cc}
1+(-1)^{m} \cos \theta_{a_{i}} & (-1)^{m} \sin \theta_{a_{i}} e^{-i \phi_{a_{i}}} \\
(-1)^{m} \sin \theta_{a_{i}} e^{i \phi_{a_{i}}} & 1-(-1)^{m} \cos \theta_{a_{i}}
\end{array}\right)
\end{aligned}
$$

is the projector of Alice for the $i$ th measurement, and similar definitions for $\hat{P}\left(b_{j}=n\right), \hat{P}\left(c_{k}=l\right)$. More precisely, for the generalized GHZ state one obtains

$$
\begin{aligned}
& P^{Q M}\left(a_{i}=m, b_{j}=n, c_{k}=l\right) \\
& =\frac{1}{8} \cos ^{2} \xi\left[1+(-1)^{m} \cos \theta_{a_{i}}\right]\left[1+(-1)^{n} \cos \theta_{b_{j}}\right]\left[1+(-1)^{l} \cos \theta_{c_{k}}\right] \\
& \left.+\frac{1}{8} \sin ^{2} \xi\left[1-(-1)^{m} \cos \theta_{a_{i}}\right]\left[1-(-1)^{n} \cos \theta_{b_{j}}\right]\left[1-(-1)^{l} \cos \theta_{c_{k}}\right)\right] \\
& +\frac{1}{8} \sin (2 \xi)(-1)^{m+n+l} \sin \theta_{a_{i}} \sin \theta_{b_{j}} \sin \theta_{c_{k}} \cos \left(\phi_{a_{i}}+\phi_{b_{j}}+\phi_{c_{k}}\right)
\end{aligned}
$$

For convenient reason, let us denote the left-hand side of the Bell inequality by $\mathcal{B}$, which represents a Bell quantity. For the following settings $\theta_{a_{1}}=\theta_{a_{2}}=\theta, \phi_{a_{1}}=-\pi / 3, \phi_{a_{2}}=2 \pi / 3, \theta_{b_{1}}=\theta_{c_{1}}=0, \phi_{b_{1}}=\phi_{c_{1}}=0, \theta_{b_{2}}=\theta_{c_{2}}=\pi / 2, \phi_{b_{2}}=$ $\phi_{c_{2}}=\pi / 6$, the Bell quantity $\mathcal{B}$ is given as $\mathcal{B}=\frac{1}{2}+\frac{3}{2}(\cos \theta+\sin (2 \xi) \sin \theta) \geq \frac{1}{2}+\frac{3}{2} \sqrt{1+\sin ^{2}(2 \xi)}$, the equal sign occurs at $\theta=\tan ^{-1}[\sin (2 \xi)]$. Obviously the Bell inequality is violated for any $\xi \neq 0$ or $\pi / 2$. This ends the proof.

This theorem indicates that it is possible for a Bell inequality in terms of probabilities to be violated by all pure entangled generalized GHZ states. Recently, classification of $N$-qubit entanglement via a quadratic Bell inequality consisting of MABK polynomials has been presented in Ref. [13. For $N=3$, there are three types of three-qubit states: i) totally separable states denoted as $\left(1_{3}\right)=\left\{\right.$ mixtures of states of form $\left.\rho_{A} \otimes \rho_{B} \otimes \rho_{C}\right\}$; ii) 2-entangled states which are denoted as $(2,1)=\left\{\right.$ mixtures of states of form $\left.\rho_{A} \otimes \rho_{B C}, \rho_{A C} \otimes \rho_{B}, \rho_{A B} \otimes \rho_{C}\right\}$; iii) fully entangled states which are denoted as $(3)=\left\{\rho_{A B C}\right\}$ including the GHZ state. Ref. 13] has drawn an ancient Chinese coin (ACC) diagram for the classification of three-qubit entanglement. However, for the four points located on the four corners of the square, some of the above three types of three-qubit states coexist. For instance, the totally separable states and the generalized GHZ states for $\xi \in(0, \pi / 12]$ coexist at these four corners, it looks somehow like these four points are "degenerate". The above Bell inequality for probabilities is useful, at least; it can distinguish the generalized GHZ states for $\xi \in(0, \pi / 12]$ from the totally separable states.

There are two different entanglement classes for three-qubit states, namely, 2-entangled states and fully entangled states. Why MABK inequalities as well as ŻB inequalities fail for the region $\xi \in(0, \pi / 12]$ may be due to the reason that their inequalities contain only fully three-particle correlations. If one expands $\hat{P}\left(a_{i}=m\right) \otimes \hat{P}\left(b_{j}=n\right) \otimes \hat{P}\left(c_{k}=l\right)$ and substitutes them into a Bell quantity $\mathcal{B}$, one will find that $\mathcal{B}$ contains not only the terms of fully three-particle correlations, such as $\hat{n}_{a_{i}} \cdot \vec{\sigma} \otimes \hat{n}_{b_{j}} \cdot \vec{\sigma} \otimes \hat{n}_{c_{l}} \cdot \vec{\sigma}$, but also the terms of two-particle correlations, such as $\hat{n}_{a_{i}} \cdot \vec{\sigma} \otimes \hat{n}_{b_{j}} \cdot \vec{\sigma} \otimes \mathbf{1}$. The above theorem implies that two-particle correlations may make a contribution to the quantum violation of Bell inequality. The remarkable property of the Bell inequality in Eq. (2) is that it is violated by all pure entangled generalized GHZ states. However, some of other pure entangled states does not violate it, such as the W state $|\psi\rangle_{W}=(|100\rangle+|010\rangle+|001\rangle) / \sqrt{3}$. The reason may be that the Bell inequality in Eq. (2) does not contain all the possible probabilities. This motivates us to introduce a Bell inequality with all possible probabilities:

$$
\begin{aligned}
& P\left(a_{1}+b_{1}+c_{1}=1\right)+2 P\left(a_{2}+b_{2}+c_{2}=1\right) \\
& +P\left(a_{1}+b_{2}+c_{2}=2\right)+P\left(a_{2}+b_{1}+c_{2}=2\right)+P\left(a_{2}+b_{2}+c_{1}=2\right) \\
& -P\left(a_{1}+b_{1}+c_{2}=0\right)-P\left(a_{1}+b_{2}+c_{1}=0\right)-P\left(a_{2}+b_{1}+c_{1}=0\right) \\
& -P\left(a_{1}+b_{1}+c_{2}=3\right)-P\left(a_{1}+b_{2}+c_{1}=3\right)-P\left(a_{2}+b_{1}+c_{1}=3\right) \leq 3 .
\end{aligned}
$$




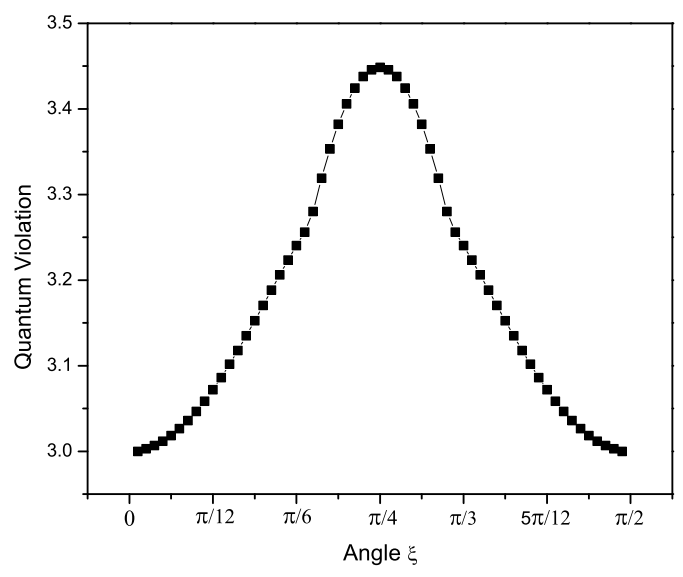

FIG. 1: Numerical results for the generalized GHZ states $|\psi\rangle_{\mathrm{GHZ}}=\cos \xi|000\rangle+\sin \xi|111\rangle$, which violate a Bell inequality for probabilities (6) except $\xi=0$ and $\pi / 2$. For the GHZ state with $\xi=\pi / 4$, the Bell quantity reaches its maximum value $\frac{3}{8}(4+3 \sqrt{3})$.

This inequality is symmetric under the permutations of three observers: Alice, Bob and Charlie. Pure states of three qubits constitute a five-parameter family, with equivalence up to local unitary transformations. This family has the representation [14]

$$
\begin{aligned}
|\psi\rangle= & \sqrt{\mu_{0}}|000\rangle+\sqrt{\mu_{1}} e^{i \phi}|100\rangle+\sqrt{\mu_{2}}|101\rangle \\
& +\sqrt{\mu_{3}}|110\rangle+\sqrt{\mu_{4}}|111\rangle
\end{aligned}
$$

with $\mu_{i} \geq 0, \sum_{i} \mu_{i}=1$ and $0 \leq \phi \leq \pi$. Numerical results show that this Bell inequality for probabilities is violated by all pure entangled states of a three-qubit system. However, it is difficult to provide an analytic proof.

In Fig. 1 we show the numerical results for the generalized GHZ states $|\psi\rangle_{\mathrm{GHZ}}=\cos \xi|000\rangle+\sin \xi|111\rangle$, which violate the above symmetric Bell inequality for probabilities except $\xi=0$ and $\pi / 2$. For the measuring angles $\theta_{a_{1}}=\theta_{a_{2}}=\theta_{b_{1}}=\theta_{b_{2}}=\theta_{c_{1}}=\theta_{c_{2}}=\pi / 2, \phi_{a_{1}}=-5 \pi / 12, \phi_{a_{2}}=\pi / 4, \phi_{b_{1}}=-5 \pi / 12, \phi_{b_{2}}=\pi / 4, \phi_{c_{1}}=-\pi / 3, \phi_{c_{2}}=\pi / 3$, all the probability terms with positive signs in the Bell inequality (6i) are equal to $\frac{3}{16}(2+\sqrt{3})$, while the terms with negative signs are equal to $\frac{1}{8}$, so the quantum violation of the Bell quantity for the GHZ state (where $\xi=\pi / 4$ ) is obtained as $6 \times \frac{3}{16}(2+\sqrt{3})-6 \times \frac{1}{8}=\frac{3}{8}(4+3 \sqrt{3})>3$. In Fig. 2 we show the numerical results for the family of generalized W states $|\psi\rangle_{W}=\sin \beta \cos \xi|100\rangle+\sin \beta \sin \xi|010\rangle+\cos \beta|001\rangle$ with the cases $\beta=\pi / 12, \pi / 6, \pi / 4, \pi / 3,5 \pi / 12$ and $\pi / 2$, which show the quantum violation of $|\psi\rangle_{W}$ except the product cases with $\beta=\pi / 2, \xi=0$ and $\pi / 2$. For the standard W state $|\psi\rangle_{W}=(|100\rangle+|010\rangle+|001\rangle) / \sqrt{3}$, the quantum violation is 3.55153 . We now proceed to present the second theorem.

Theorem 2: All pure 2-entangled states of a three-qubit system violate a Bell inequality for probabilities.

Proof: By pure 2-entangled states of three-qubit system, we mean $\left|\psi_{A B}\right\rangle \otimes\left|\psi_{C}\right\rangle,\left|\psi_{A C}\right\rangle \otimes\left|\psi_{B}\right\rangle$ and $\left|\psi_{B C}\right\rangle \otimes\left|\psi_{A}\right\rangle$. It is sufficient to consider one of them, say $\left|\psi_{A B}\right\rangle \otimes\left|\psi_{C}\right\rangle$, since the Bell inequality (6) is symmetric under the permutations of $A, B$ and $C$. Moreover, one can always have $\left|\psi_{A B}\right\rangle \otimes\left|\psi_{C}\right\rangle=\left(\cos \xi|00\rangle_{A B}+\sin \xi|11\rangle_{A B}\right) \otimes|0\rangle_{C}$ due to local unitary transformations. For the measuring angles $\theta_{a_{1}}=\theta_{a_{2}}=\theta, \phi_{a_{1}}=2 \pi / 3, \phi_{a_{2}}=-\pi / 3, \theta_{b_{1}}=\theta_{c_{1}}=0, \phi_{b_{1}}=$ $\phi_{c_{1}}=0, \theta_{b_{2}}=\pi / 2, \theta_{c_{2}}=\pi, \phi_{b_{2}}=\pi / 3, \phi_{c_{2}}=0$, we obtain from the left-hand side of the Bell inequality (6) that $\mathcal{B}=\frac{3}{2}(1-\cos \theta+\sin (2 \xi) \sin \theta) \geq \frac{3}{2}\left(1+\sqrt{1+\sin ^{2}(2 \xi)}\right)$, the equal sign occurs at $\theta=-\tan ^{-1}[\sin (2 \xi)]$. Obviously the Bell inequality is violated for any $\xi \neq 0$ or $\pi / 2$. This ends the proof. Indeed, the quantum violation of the state $\left|\psi_{A B}\right\rangle \otimes\left|\psi_{C}\right\rangle$ corresponds to the curve with $\beta=\pi / 2$ as shown in Fig.2, because $\left|\psi_{A B}\right\rangle \otimes\left|\psi_{C}\right\rangle$ is equivalent to $|\psi\rangle_{W}$ for $\beta=\pi / 2$ up to a local unitary transformation.

There is a simpler and more intuitive way to prove Theorem 2, because the symmetric Bell inequality (6) can be reduced to a CHSH-like inequality for two qubits and then from Gisin's theorem for two qubits, one easily has 


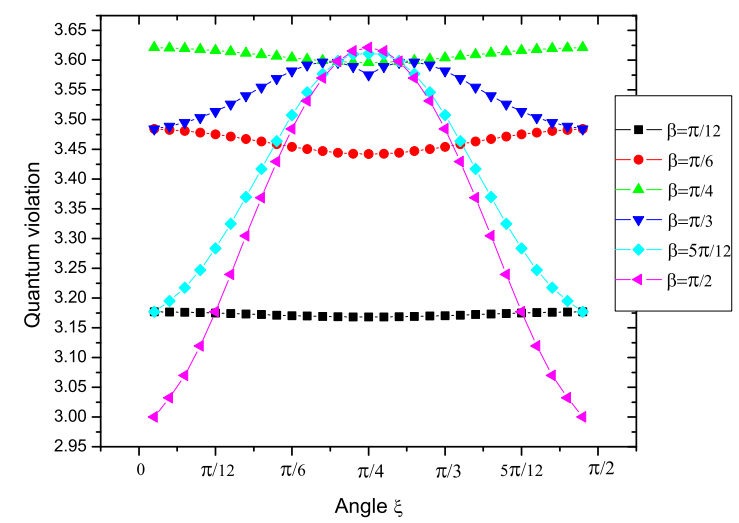

FIG. 2: Numerical results for the family of generalized W states $|\psi\rangle_{W}=\sin \beta \cos \xi|100\rangle+\sin \beta \sin \xi|010\rangle+\cos \beta|001\rangle$ with the cases $\beta=\pi / 12, \pi / 6, \pi / 4, \pi / 3,5 \pi / 12$ and $\pi / 2$.

Theorem 2. By taking $c_{1}=0, c_{2}=1$, we have from Eq. (6) that

$$
\begin{aligned}
& P\left(a_{1}+b_{1}=1\right)+2 P\left(a_{2}+b_{2}=0\right) \\
& +P\left(a_{1}+b_{2}=1\right)+P\left(a_{2}+b_{1}=1\right)+P\left(a_{2}+b_{2}=2\right) \\
& -P\left(a_{1}+b_{1}=-1\right)-P\left(a_{1}+b_{2}=0\right)-P\left(a_{2}+b_{1}=0\right) \\
& -P\left(a_{1}+b_{1}=2\right)-P\left(a_{1}+b_{2}=3\right)-P\left(a_{2}+b_{1}=3\right) \leq 3
\end{aligned}
$$

Since $a_{1}, a_{2}, b_{1}, b_{2}=0,1$, the probabilities $P\left(a_{1}+b_{1}=-1\right), P\left(a_{1}+b_{2}=3\right)$ and $P\left(a_{2}+b_{1}=3\right)$ will be equal to zero, by using $P\left(a_{2}+b_{2}=0\right)+P\left(a_{2}+b_{2}=2\right)=1-P\left(a_{2}+b_{2}=1\right)$, we arrive at the following Bell inequality for two-qubit:

$$
\begin{aligned}
& P\left(a_{1}+b_{1}=1\right)+P\left(a_{1}+b_{2}=1\right)+P\left(a_{2}+b_{1}=1\right)+P\left(a_{2}+b_{2}=0\right) \\
& -P\left(a_{1}+b_{1}=2\right)-P\left(a_{1}+b_{2}=0\right)-P\left(a_{2}+b_{1}=0\right)-P\left(a_{2}+b_{2}=1\right) \leq 2 .
\end{aligned}
$$

This Bell inequality is symmetric under the permutations of Alice and Bob, it is an alternative form for CHSH inequality of two qubits. For the two-qubit state $|\psi\rangle=\cos \xi|00\rangle+\sin \xi|11\rangle$ and the projector as shown in Eq.(44), one can have the quantum probability $P^{Q M}\left(a_{i}=m, b_{j}=n\right)=\frac{1}{4} \cos ^{2} \xi\left[1+(-1)^{m} \cos \theta_{a_{i}}\right]\left[1+(-1)^{n} \cos \theta_{b_{j}}\right]+$ $\frac{1}{4} \sin ^{2} \xi\left[1-(-1)^{m} \cos \theta_{a_{i}}\right]\left[1-(-1)^{n} \cos \theta_{b_{j}}\right]+\frac{1}{4} \sin (2 \xi)(-1)^{m+n} \sin \theta_{a_{i}} \sin \theta_{b_{j}} \cos \left(\phi_{a_{i}}+\phi_{b_{j}}\right)$. For the measuring angles $\theta_{a_{1}}=\theta_{a_{2}}=\theta, \phi_{a_{1}}=\pi-\phi, \phi_{a_{2}}=-\phi, \theta_{b_{1}}=0, \phi_{b_{1}}=0, \theta_{b_{2}}=\pi / 2, \phi_{b_{2}}=\phi$, the left-hand side of a Bell inequality (9) becomes $\mathcal{B}=\frac{1}{2}+\frac{3}{2}(-\cos \theta+\sin (2 \xi) \sin \theta) \geq \frac{1}{2}\left(1+3 \sqrt{1+\sin ^{2}(2 \xi)}\right)$, the equal sign occurs at $\theta=-\tan ^{-1}[\sin (2 \xi)]$. Obviously a Bell inequality (9) is violated for any $\xi \neq 0$ or $\frac{\pi}{2}$, just the same as CHSH inequality violated by the twoqubit state $|\psi\rangle=\cos \xi|00\rangle+\sin \xi|11\rangle$. For the Werner state $\rho_{W}=V|\psi\rangle\langle\psi|+(1-V) \rho_{\text {noise, }}$, where $|\psi\rangle=(|00\rangle+|11\rangle) / \sqrt{2}$ is the maximally entangled state. The maximal value of $V$ that a local realism is still possible by this Bell inequality is $V_{\max }=1 / \sqrt{2}$, just the same as the case for CHSH inequality. Actually, if one denotes the left-hand side of Bell inequality (9) by $\mathcal{B}$ and redefines a new Bell quantity $\mathcal{B}^{\prime}=\frac{4}{3}\left(\mathcal{B}-\frac{1}{2}\right)$, he still has a Bell inequality $\mathcal{B}^{\prime} \leq 2$. For quantum mechanics, $\mathcal{B}_{\max }^{\prime}=2 \sqrt{1+\sin ^{2}(2 \xi)}$, which reaches $2 \sqrt{2}$ and then $\mathcal{B}^{\prime}$ recovers the usual CHSH inequality.

Theorem 2 is remarkable. If one knows that a pure state is a 2-entangled state of a three-qubit system, one can use a Bell inequality (6) to measure the degree of entanglement (or concurrence denoted by $\mathcal{C}$ ) of the state. Since $\mathcal{B}_{\max }=\frac{3}{2}\left(1+\sqrt{1+\sin ^{2}(2 \xi)}\right)=\frac{3}{2}\left(1+\sqrt{1+\mathcal{C}^{2}}\right)$, thus one has the concurrence $\mathcal{C}=|\sin (2 \xi)| \in[0,1]$, just the same as the case of CHSH inequality measure of the concurrence of pure states of two qubits. In summary, (i) since all pure entangled states (including pure 2-entangled states) of a three-qubit system violate a Bell inequality (6), thus we have Gisin's theorem for a three-qubit system; (ii) the Bell inequality (6) can be reduced to an alternative form of the CHSH inequality (in terms of probabilities), thus it can be viewed as a good candidate for a "natural" generalization of the usual CHSH inequality. (iii) MABK inequalities and ŻB inequalities are binary correlation Bell inequalities. However, 
one may notice that Bell inequalities (2) and (6) are both ternary Bell inequalities, i.e., where the inequalities are "modulo 3". Most recently, a ternary Bell inequality in terms of probabilities for three qutrits was presented in Ref. [15], this inequality can be connected to the Bell inequality (6), which is for three qubits if one restricts the initial three possible outcomes of each measurement to only two possible outcomes.

We thank M. Żukowski for valuable discussion. This work is supported by NUS academic research Grant No. WBS: R-144-000-089-112. J.L. Chen acknowledges financial support from Singapore Millennium Foundation and (in part) by NSF of China (Grant No. 10201015). C. Wu acknowledges financial support by NUS.

* Electronic address: phycj@nus.edu.sg

$\dagger$ Electronic address: phyohch@nus.edu.sg

[1] J. S. Bell, Physics (Long Island City, N.Y.) 1, 195 (1964).

[2] J. Clauser, M. Horne, A. Shimony, and R. Holt, Phys. Rev. Lett. 23, 880 (1969).

[3] D. M. Greenberger, M. Horne, A. Shimony, and A. Zeilinger, Am. J. Phys. 58, 1131 (1990).

[4] N. D. Mermin, Phys. Rev. Lett. 65, 1838 (1990); M. Ardehali, Phys. Rev. A 46, 5375 (1992); A. V. Belinskii and D. N. Klyshko, Phys. Usp. 36, 653 (1993).

[5] J.L. Chen, D. Kaszlikowski, L.C. Kwek, and C.H. Oh, Mod. Phys. Lett. A 17, 2231 (2002); J.L. Chen, D. Kaszlikowski, L.C. Kwek, C.H. Oh, and M. Żukowski, Phys. Rev. A 64, 052109 (2001); D. Kaszlikowski, L.C. Kwek, J.L. Chen, M. Żukowski, and C.H. Oh Phys. Rev. A 65, 032118 (2002); L.B. Fu, J.L. Chen, and X.G. Zhao, Phys. Rev. A 68, 022323 (2003). L.B. Fu, Phys. Rev. Lett. 92, 130404 (2004); D. Kaszlikowski, D. K. L. Oi, M. Christandl, K. Chang, A. Ekert, L.C. Kwek, and C.H. Oh, Phys. Rev. A 67, 012310 (2003); D. Collins, N. Gisin, N. Linden, S. Massar, and S. Popescu, Phys. Rev. Lett. 88, 040404 (2002).

[6] N. Gisin, Phys. Lett. A 154, 201 (1991); N. Gisin and A. Peres, Phys. Lett. A 162, 15-17 (1992).

[7] S. Popescu and D. Rohrlich, Phys. Lett. A 166, 293 (1992).

[8] V. Scarani and N. Gisin, J. Phys. A 346043 (2001).

[9] M. Żukowski, Č. Brukner, W. Laskowski, and M. Wiesniak, Phys. Rev. Lett. 88, 210402 (2002).

[10] M. Żukowski and Č. Brukner, Phys. Rev. Lett. 88, 210401 (2002).

[11] H. Weinfurter and M. Żukowski, Phys. Rev. A 64, 010102(R) (2001).

[12] R. F. Werner and M. M. Wolf, Phys. Rev. A 64, 032112 (2001).

[13] S. Yu, Z.B. Chen, J.W. Pan, and Y.D. Zhang, Phys. Rev. Lett. 90, 080401 (2003).

[14] A. Acín, A. Andrianov, L. Costa, E. Jané, J. I. Latorre, and R. Tarrach, Phys. Rev. Lett. 85, 1560 (2000); A. Acín, A. Andrianov, E. Jané, and R. Tarrach, J. Phys. A 34, 6725 (2001);C. Emary and C.W.J. Beenakker, Phys. Rev. A 69, 032317 (2004).

[15] A. Acín, J.L. Chen, N. Gisin, D. Kaszlikowski, L.C. Kwek, C.H. Oh, and M. Żukowski, Phys. Rev. Lett. 92, 250404 (2004). 